\title{
ZUR AGRARGEOGRAPHIE DES VORDERRHEINTALES
}

\author{
Casper Peter Casutt und Emil Rauch
}

Die schweizerische Gebirgslandwirtschaft steht wie die mittelländische in einem Umbruch, der auch den Charakter der Landschaft wesentlich beeinflussen wird. Auch die Geographie hat deshalb diesen Vorgängen ihr Interesse zu schenken. In diesem Zusammenhang verdienen Erhebungen der Schweizerischen Vereinigung zur Förderung der Betriebsberatung in der Landwirtschaft besondere Aufmerksamkeit, weil sie gerade für die Erfassung der Agrarlandschaft wertvolle Hinweise bieten. Im folgenden seien daher die Ergebnisse einer Enquete im bündnerischen Vorderrheintal mitgeteilt. (Die Redaktion).

\section{METHODISCHES}

Die Schweizerische Vereinigung zur Förderung der Betriebsberatung in der Landwirtschaft hat im Rahmen der ihr gestellten Aufgaben, insbesondere dem Berggebiet ihre Aufmerksamkeit geschenkt. Etwa 10000 Bergbetriebe sind in kleinen Beratungsgruppen zusammengeschlossen und die von jedem einzelnen Betrieb erstellten Betriebsspiegel geben den von den Kantonen eingesetzten Beratern die Möglichkeit, durch Betriebsvergleiche festzustellen, welche Betriebe und in welcher Beziehung dieselben einer Beratung bedürfen. Trotzdem den ersten Erhebungen noch verschiedene Entwicklungsmängel anhaften und vor Schlußfolgerungen, die ins Einzelne gehen, gewarnt werden muß, geben die Daten aus dem Bündner Oberland, die sich auf die Kontrolle, Interpretation und Zielsetzung des seit seiner Jugend mit dem Gebiet verwachsenen Beraters (C. P. CASUTT) stützen, die Möglichkeit einer zuverläßigen Beurteilung des Gebietes. Aus den sechs Kreisen Trins, Disentis, Ruis, Lugnez, Ilanz und Safien, sowie aus der Gemeinde Rhäzüns, die nach der Betriebszählung 19553162 landwirtschaftliche Betriebe umfassen, liegen 1700 Betriebsspiegel vor, so daß über $50 \%$ der Güter untersucht sind. Die Enquête darf daher als repräsentativ gelten. Betriebe mit weniger als zwei Großvieheinheiten sind darin nicht enthalten, weil bei ihnen schon kleine Erhebungsfehler die Ergebnisse stark entstellen können. Der Anschluß an eine Beratungsgruppe ist aber trotzdem allen Kleinbauern möglich.

Der Betriebsspiegel für Berggebiete ist ein Fragebogen, der die Betriebsanalyse ermöglichen soll. Er hält zunächst den Betriebsumfang, seine Unterteilung nach Talgut, Maiensäss, Ausfütterungsställen, Vorwinterungen, Alpen, die Parzellierung, Gebäudezahl und Besitzart fest. Sodann erfaßt er die Grasnutzung und deren Futterleistung nach Fett- und Magerwiese, Herbst-, Frühjahrs- und Vorweide, ihren Standort, den Acker- und Gartenbau nach Fruchtart, Ertrag und dem Zweck (Selbstversorgung, Verfütterung und der Verkauf). Die Viehhaltung wird nach Art und Umfang, nach Alter, Sömmerungszeit, Kalbetermin, Produktionsrichtung (Milch, Fleisch, Nachzucht) und nach ihrer Leistung untersucht. Bei den Maschinen müssen Zug- und Antriebsmaschinen, Traktoren, Einachser, Motormäher, Seilwinden nach PS, aber auch stationäre Maschinen zum Pumpen, Holzsägen usw. nach ihrem Wert, Alter und Einsatz festgehalten werden. Dasselbe gilt für die angetriebenen Arbeitsmaschinen und andere Geräte mehr. Daneben ist es aber nötig, die Mengen an zugekauftem Kraftfutter, Heu, Stroh, Kunstdünger usw. zu kennen, die den direkten Aufwand erhöhen. Nicht zuletzt ist die auf dem Betrieb tätige Arbeitskraft festzustellen, mit der Produktionsleistung des Betriebes zu vergleichen, wobei die Arbeit für Dritte, die Arbeitshilfe durch Dritte, sowie der Nebenerwerb in der Produktionsrechnung berücksichtigt werden müssen.

Auf Grund dieser Angaben werden Lochkarten hergestellt, die auf einer elektronischen Rechenmaschine verarbeitet werden. Die Ergebnisse werden für jede Gruppe (meist eine Gemeinde umfassend), zusammengestellt und können dann mit den Betriebsleitern diskutiert werden. Durchschnittswerte werden vermieden; die Spanne, in der mindestens $50 \%$ der beteiligten Betriebe liegen, ist wichtiger. In anderen Fällen genügt es festzustellen, ob eine Güllengrube vorhanden ist, ob Stroh zugekauft wird etc. Wir ersehen im Titel der Erhebungstabellen die Regional- und Gruppenzahl, sodann als erste Spalte die Nummer des Betriebes, die dem Einzelnen zwar seine Ergebnisse zeigt, die jedoch den Namen der andern Betriebe verschweigt. In folgenden Spalten sind verschiedene Schlüsselzahlen der Betriebe in Vergleich gestellt. Es zeigen:

Spalte 2: Die Hauptfutterfäche je Großvieheinheit und damit die Produktivität des Bodens.

Spalte 3: Den Fettwiesen-Anteil an der gesamten Grünlandfläche.

Spalte 4: Den vom Talbetrieb gelieferten Anteil an der gesamten Futterproduktion.

Spalte 5: Die Durchschnitts-Milchleistung je Kuh in 100 Kilogramm.

Spalte 6: Den Verkehrsmilchanteil.

Spalte 7: Den Kraftfutterzukauf je Rinder-Großvieheinheit (GVE). 
Spalte 8: Den Rindvieh-Anteil am gesamten Tierbestand (Pferde, Ziegen, Schafe).

Spalte 9: Den Düngerzukauf je Rinder-GVE (wichtiger als je ha).

Spalte 10: Den Heuhandel.

Spalte 11: Den Strohzukauf.

Spalte 12: Die Arbeitstage je GVE.

Damit ist die Auswertung des Spiegels keinesfalls erschöpft. Weitere Zusammenstellungen zeigen andere Betriebsprobleme. Das Gruppenblatt deutet dem Berater die schwachen Betriebe oder richtiger die Betriebsschwäche an. Erst die Prüfung, warum einzelne Betriebe ungünstigere Ergebnisse haben als die Mittelgruppe und warum einzelne Betriebe besser dastehen, darf zu Schlußfolgerungen führen. Der Spiegel ist keineswegs die einzige Untersuchung, sondern es werden neben demselben weitere Erhebungen nötig, um spezielle Probleme, sei es die Mechanisierung oder die Arbeitsrationalisierung usw. abzuklären. Um regionale Vor- und Nachteile des Klimas, des Bodens, der Siedlungsstruktur, der Betriebsausrüstung usw. zu erkennen, müssen die Gruppen der einzelnen Kreise und die Ergebnisse aus den sechs Kreisen untereinander, ja mit den Zahlen aus anderen Kantonen verglichen werden. Dabei ist soweit als möglich die politische Region durch eine klimatische usw. zu ersetzen. In unserem Gebiet ist zu beobachten, daß lediglich der Umfang des Ackerbaues regional differenziert ist. Klimatische und topographische Voraussetzungen sind dafür ausschlaggebend. Alle übrigen Ergebnisse weisen innerhalb der einzelnen Gruppen größere Unterschiede auf als die Mittelzahlen der Gruppen und Kreise, ein Beweis für die Bedeutung der Kenntnisse des Bewirtschafters und damit für die Notwendigkeit und Nützlichkeit der Beratung.

Im Rahmen dieser Arbeit war es dagegen nicht möglich, den Einfluß der Höhenlage und anderer natürlicher Einflüsse auf den Ertrag des Graslandes und auf den Charakter der Betriebe nach Regionen zu trennen.

\section{DAS GEBIET}

Das Vorderrheintal ist die Region des gleichnamigen Flusses bis zur Mündung des Hinterrheins. Sie wird im Westen durch das östliche Gotthardmassiv (Wasserscheide Rhein-Tessin-Reußtal), im Norden durch die von steilen Tälern zerschnittene Südkette der Glarneralpen, im Süden durch den Südrand der Adula-Alpen (Hinter. rheintal) begrenzt. Der bündnerische Bezirk Vorderrhein umfaßt nur einen guten Drittel des natürlichen Einzugsgebietes von $1515 \mathrm{~km}^{2}$. Diesem gehören noch die Bezirke Imboden, Glenner und der Kreis Safien an. Von rund $2500 \mathrm{~m}$ (Tomasee am Badus $2341 \mathrm{~m}$ ) senkt sich das Haupttal über eine Strecke von gut $70 \mathrm{~km}$ (mittleres Gefälle $25 \%$ ) auf rund $580 \mathrm{~m}$ (Reichenau). Auch Oberland genannt, stellt es eine ausgesprochene Terrassenlandschaft dar, die durch den späteiszeitlichen, stark bewaldeten Bergsturz von Flims in die kleinere Talschaft Sutselva (Nid dem Wald) und die geräumige Surselva ( $\mathrm{Ob}$ dem Wald), das eigentliche Bündner Oberland, gegliedert wird. Die Nordseite des Tals ist teils (Westhälfte) in Gneise, Granite, und Diorite des Gotthardmassivs, teils (Osthälfte) in Sedimente, Schiefer, Konglomerate und Kalke des Verrukanos, der Juraformation und des Eozäns hineinmodelliert und trägt firngekrönte Gipfel (Oberalpstock 3327 m, Tödi 3623 m, Piz Segnes 3102 m, Ringclspitz $3251 \mathrm{~m}$ ). Die südlichen, durch längere Quertäler (Val Medels, Sumvitg, Lungnez, Safien) gegliederten Gebiete, gehören vornehmlich (mit Ausnahme der kristallinen Massen des südöstlichen Gotthardmassivs und der nördlichen Adulaecke (Rheinwaldgebiet) schiefrigen Gesteinen (Bündnerschiefern) der unter- u. mittelpenninischen Decken an. Ihre Umrahmung überragen (Piz Beverin $3002 \mathrm{~m}$ ) nicht minder majestätische Berghäupter. Die Böden wechseln daher ebenso oft wie das vielgestaltige Relief und die Gesteine, wobei jedoch in zahlreiche Becken gegliederte Schutthügellandschaften durchaus vorherrschen. $\mathrm{Da}$ das Vorderrheintal im ausgesprochenen Regenschatten liegt, ist es klimatisch eine begünstigte Insel, der relative Trockenheit (Talgrund $80-150$, Höhen bis $250 \mathrm{~cm}$ Niederschlag) und verhältnismäßig temperierte Wärmeverhältnisse (Talgründe: Januar -5 bis -2 , Juli $15-18^{\circ}$; Höhen allerdings größere Kühle: Januar $-10^{\circ}$, Juli $5^{\circ}$ ) eignen. Vor der «großen» Rodung und Besiedlung des Gebietes von XIII. bis zum XVI. Jahrhundert war es daher bis etwa $2000 \mathrm{~m}$ (wie Arvenreste etwa auf der Alp Nalps und Toma $2020 \mathrm{~m}$ bezeugen) ein ausgesprochenes Waldgebirgstal. Ihm geben Fichte (noch jetzt bis $75 \%$ der Baumbestände), Weißtannen, Waldföhren und Legföhren, Lärchen und Arven das Gepräge. 


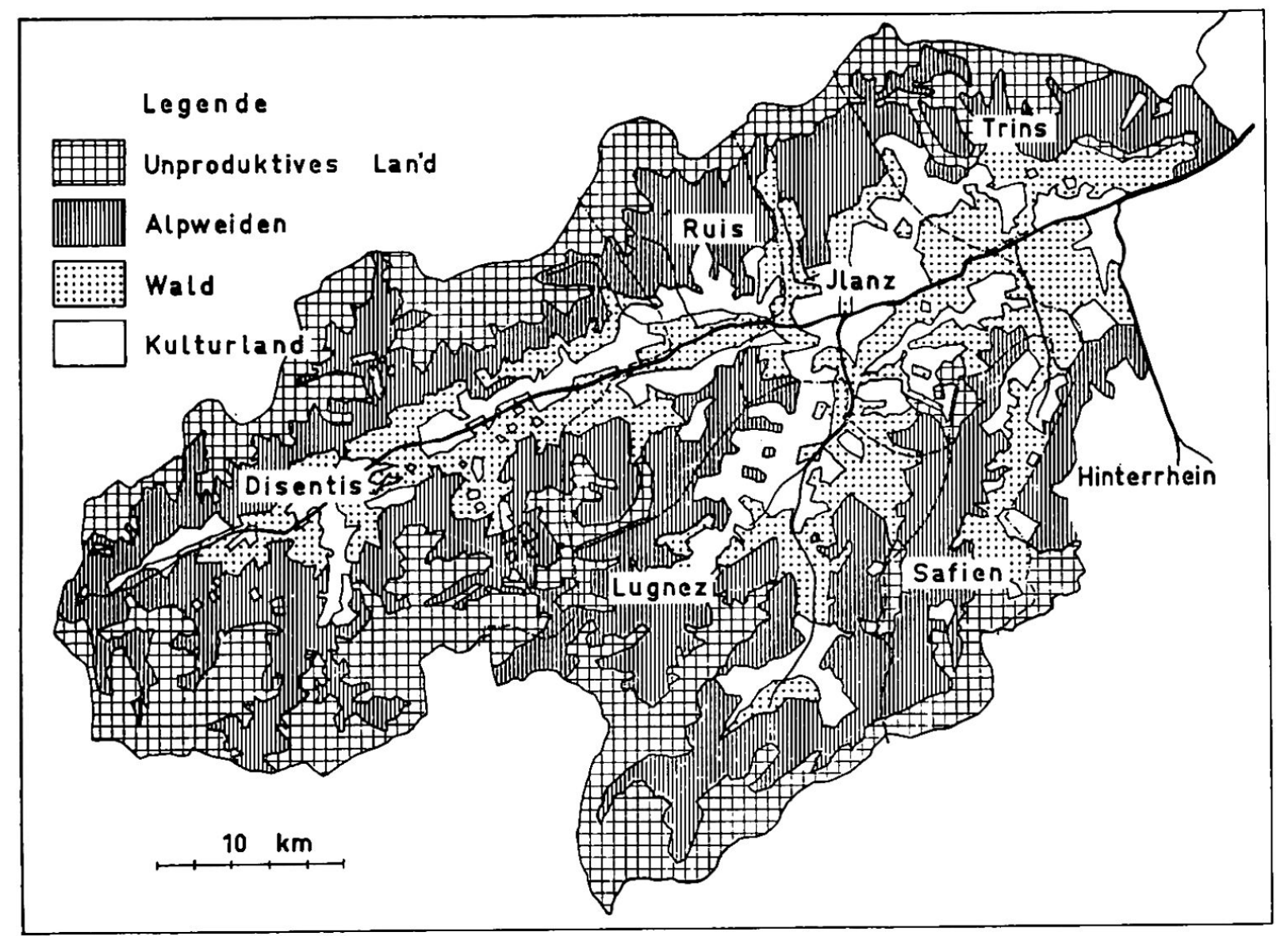

Agrargeographische Skizze des Vorderrheintales (H. Hotz)

In römischer Zeit der Raetia prima angehörig, kam das Oberland in der Folge ans Fränkische Reich, dessen politische Gliederungen in Gaus und Zehnten noch in den heutigen Bezirken und Kreisen Ausdruck finden. Die vornehmlich rätische Bevölkerung mit breiten Schädeln, braunen Augen und dunkeln Haaren pflegt seit alters Acker-, Garten-, Obst- und Grasbau, den immerhin im ganzen eher kärglichen Naturbedingungen entsprechend in teils geschlossenen Dorf- und Weiler-, teils in weit zerstreuten Hofsiedlungen und nur langsam wachsender Gesamtzahl (1850: 23 989, 1960: 32 267, + 34\%). Die Dichte liegt daher auch jetzt noch, (mit Ausnahmen: Ilanz bis Flims 70-100 je $\mathrm{km}^{2}$ ) wesentlich unter dem schweizerischen Mittel von etwa 120 (10-25, in den Hochlagen unter 10). Obwohl im 19. Jahrhundert eine durchgehende Eisenbahn Chur-Martigny die Talschaft der «großen Welt» erschloß, nachdem sie schon früher einen regen Träger- und Saumverkehr (Lukmanier, Oberalp usw.) unterhalten hatte, blieb sie, von wenigen Fremdenverkehrszentren (Flims, Sedrun), Elektrizitätswerken, Fabrikbetrieben abgesehen, eine ausgesprochene alpine Agrarlandschaft.

\section{DIE AGRARLANDSCHAFT}

Der eidg. Viehwirtschaftskataster teilt den größten Teil des Gebietes seiner ungünstigsten Zone 3, dagegen die Gemeinden Castrisch, Ilanz, Sagens, Schluein, Trin, Valendas und Versam der Zone 2 zu. Nur die Gemeinde Rhäzüns rechnet er zur wirtschaftlich günstigsten Zone 1. Von der Gesamtfläche von 153927 ha sind 44209 ha unproduktiv, 27010 ha Wald und 82708 ha Nutzfläche ohne Wald. In eigener Bewirtschaftung stehen ca. 17000 ha (geschätzte Fläche auf Grund der mittleren Betriebsgröße und der Zahl der Landwirtschaftsbetriebe nach der Betriebszählung 1955), d.h. etwa ein Fünftel des landwirtschaftlichen Kulturlandes. Vier Fünftel, näm. lich die Alpen und Weiden werden seit Jahrhunderten gemeinschaftlich genutzt. Der Wald ist ebenfalls meist Gemeinde- seltener Korporationswald. 
Die bestockte Waldfläche von 22323 ha wies 1959 einen Hiebsatz von je 2,9 fm. Holz je ha auf und brachte 1959 etwa Fr. 80.- Reinertrag je fm., das sind 5,1 Mio Franken Ertrag, der namentlich öffentlichen Aufgaben dient. Den bisher unterstützten Gemeinden: zehn im Kreis Lugnez, drei im Kreis llanz und eine im Kreis Ruis dürfte der Ausbau der neuen u. der projektierten Elektrizitätswerke wesentlich helfen. 182 Primar- und 44 Sekundarschulen werden durch 91 Arbeitsschulen, 1 Gewerbeschule, eine Bäuerinnenschule und die Klosterschule in Disentis (Maturareife) ergänzt. 1955 wurden 553 Gewerbe- und Industriebetriebe mit 3562 Beschäftigten gezählt.

Das Ackerland erreicht nur selten $10 \%$ der privatwirtschaftlich genutzten Fläche. Das Sommerfutter (Weide) übersteigt demnach den Winterbedarf. So wird Sömmerungsvieh in Alpung genommen. 1956 wurden rund 21000 rauhfutterverzehrende Großvieheinheiten (ohne Schweine) in dem Gebiete gehalten. Der geringe Anteil der Kühe am Rinderbestand (33\%) ist ein Zeichen dafür, daß die Produktionsrichtung in der Viehhaltung in der Aufzucht von Jungtieren und nicht von Milch- oder Masttieren liegt. 79 Viehzuchtgenossenschaften mit 10000 punktierten Herdbuchtieren sorgen für eine fortschrittliche Zucht der einheitlichen Braunviehrasse. Der Durchschnitt der Betriebe ereicht nur etwa 5 ha (ohne Alpen). Dies führte frühzeitig zum Nebencrwerb, neuerdings zur Aufgabe der Landwirtschaft, zur Abwanderung aus dem Betrieb und zur Vergrößerung der verbleibenden Betriebe. Von den 3406 landwirtschaftlichen Betrieben im Jahre 1939 bestanden 1955 noch 3162. Während 19392569 Betriebe hauptberuflich geführt wurden, waren es 1955 noch 2242. Es erfolgte also eine Vergrößerung des Durchschnittbetriebes; sie wird aber erst zum Erfolg führen, wenn die Parzellierung verschwindet und andere Strukturmängel behoben werden.

Zur vollen Ausnutzung der Arbeitskraft in den Familienbetrieben müssen Nebeneinnahmen, sei es aus Fremdenbeherbergung, aus Nebenberufen, durch auswärts tätige Familienmitglieder, aus Waldarbeiten im Winter und anderen Möglichkeiten treten. Grundlage der Sanierung sind Zusammenlegung, Vergrößerung und Rationalisierung des landwirtschaftlichen Betriebes. Daneben hat die gewerbliche Durchdringung zur Kräftigung des Gemeindewesens und damit trotz des Rückganges der landwirtschaftlichen Bevölkerung zur Erhaltung einer volkswirtschaftlich erwünschten Volksdichte zu schreiten.

Die Erträge der landwirtschaftlichen Betriebe des Vorderrheintales entstammen zu über $90 \%$ der Rindviehhaltung und bei dieser größtenteils dem Verkauf von Zuchtund Nutztieren oder Mastkälbern. Der Milchverwertung sollte neben der Selbstversorgung mit Milch und der Verfütterung von Milch vermehrte Bedeutung geschenkt werden. Die Milchleistungen von durchschnittlich $2725 \mathrm{~kg}$ Milch je Kuh bei einem Fettgehalt von ca. $4 \%$ stehen den Zuchtergebnissen anderer Gebiete nicht nach. Der abnehmende Bedarf an Zuchttieren und die verschärfte Konkurrenz zwingen zur sorgfältigen Auslese und werden dem Schlachtviehverkauf (Mastkälber) vermehrte Bedeutung bringen. Bei der ersten Betriebsdurchleuchtung war es leider unmöglich, ein Bild über die Einnahmen aus den verschiedenen Nutzungen des Rindviehbestandes zu gewinnen. Immerhin zeigte sich, daß die Stärke der Landwirte des Vorderrheintales auf züchterischem Gebiet liegt und daß die Beratung deshalb stärker auf Fragen des Futterbaus, der Fütterung, der Düngung und der Betriebsorganisation eingehen mu? als auf die rein züchterischen. An Futterfläche werden pro Großvieheinheit zwischen 60 und 170 Aren benötigt. Der Futterertrag ist im Berggebiet durch Höhenlage, klimatische Exponiertheit und durch die Düngung so verschieden, daß eine allgemeine Erhebung nicht ausreicht, festzustellen, wie weit die natürlichen Verhältnisse und die Bewirtschaftung zu den niedrigen Erträgen führte. Sicher sind jedoch die Erträge der Wiesen und Weiden durch verbesserte Pflege und Düngung zu wesentlicher Leistungssteigerung fähig. Eine verbesserte Betriebsstruktur ist hierfür Voraussetzung, vor allem zur Selbstversorgung. 
Der Ackerbau spielt im Vorderrheintal neben der Viehhaltung eine berechtigte Rolle. $82 \%$ der untersuchten Betriebe pflanzen Getreide, 94\% Kartoffeln. Die Getreideund Kartoffelerträge sind nach den Erhebungen gut. Doch ist die Streuung zwischen den guten und schlechten Erträgen innerhalb ein- und derselben Gemeinde so groß, daß es zu den wichtigsten Zielen der Betriebsberatung gehören wird, auf diesem Produktionszweig die Technik durch besseres Saatgut, bessere Bodenbearbeitung, Unkrautbekämpfung, Düngung und Pflege zu heben und dadurch gleichmäßigere, höhere und nach Klima und Boden erzielbare Erträge zu sichern.

Nachfolgende Tabelle zeigt den Vergleich von Erträgen im schweizerischen Mittel und der im Vorderrheintal festgestellten Leistungen.

$\begin{array}{lcc}\text { Bei den folgenden Früchten umfasste die } & \text { Getreide } & \text { Kartoffeln } \\ \text { Mittelgruppe } & 64,6 \% & 57,6 \% \\ \text { Der Ertrag lag zwischen } & 10-25 \mathrm{q} & 170-300 \mathrm{q} \\ \text { Höhere Erträge wurden erzielt von } & 10,6 \% & 16,8 \% \\ \text { Niedrigere Erträge wurden erzielt von } & 23,8 \% & 25,6 \% \\ \text { Schweizerische Mittelzahl } & 26 \mathrm{q} & 230 \mathrm{q}\end{array}$

Diese ackerbaulichen Erträge sind allerdings mit einem höheren Arbeitsaufwand belastet als die Erzeugnisse im Flachland.

Sicher können Weizen und Mais, also Getreidesorten mit hohen und wertvollen Erträgen nicht angebaut werden. Trotzdem beweisen die Ergebnisse von Roggen und Gerste, daß der Ackerbau im Vorderrheingebiet stärkere Beachtung verdient und im Interesse einer besseren Betriebsform, Arbeitsverteilung, also Ausnützung der Arbeitskraft usw. an Ausdehnung zunehmen könnte. Daß der Saatkartoffelbau mit seinen höheren Preisen in hierzu geeigneten Lagen der bergbäuerlichen Landwirtschaft zusätzliche Einnahmen bringen kann, sei in diesem Bericht nur am Rande vermerkt.

Beim A ufwand fällt auf, daß $29 \%$ der Landwirte keinen Kunstdüngerzukauf haben. Aber auch die 71\%, die Kunstdünger verwenden, verbrauchen nur Fr. 11.07 je Großvieheinheit. Das ist umso bedeutungsvoller, wenn man berücksichtigt, wie mangelhaft der eigene Hofdünger wegen der ungünstigen Betriebsstruktur, ausgenützt werden kann. Die Magerwiesen und der schlechte Pflanzenbestand des übrigen Graslandes beweisen den Nährstoffmangel. Eine verbesserte Nutzung des Stalldüngers kann erst nach der Zusammenlegung unter Konzentration auf wenige Stallungen des einzelnen Betriebes erreicht werden. Bis zur Verbesserung der Betriebsstruktur muß deshalb der Kunstdünger für den Nährstoffersatz sorgen. Der Aufwand für Streustroh ist etwas höher als für Kunstdünger. Die Landwirte sind also an einer verbesserten Stalldüngernutzung interessiert. Kraftfutterzukäufe sind im allgemeinen üblich. Sie werden von $95 \%$ der Landwirte getätigt. Der hierfür aufgewendete Betrag von Fr. 30.- pro GVE liegt kaum niedriger als in anderen Regionen. Einsparungen sollten möglich sein, trotzdem der Landwirt in vermehrtem Maße nach einer leistungsangepaßten Fütterung streben muß. Verbesserte Heuqualität, Silos, Eigenanbau von Gerste können dazu beitragen. Der Heuzukauf wurde zwar nur von $14 \%$ der untersuchten Betriebe getätigt, beträgt jedoch bei den betr. Betrieben Fr. 33.25 je GVE, 10\% der Dürrfutterkosten. $\mathrm{Da}$ die kaufenden Betriebe in allen Betriebsklassen anzutreffen sind, ist anzunehmen, da $\beta$ es sich weniger um regelmäßige Zukäufe (Bahnhofbauern!) oder um Aufstokkungskäufe kleinerer Betriebe handelt als darum, die in einzelnen Jahren fehlenden Rauhfuttermengen zu decken. Trotzdem weist die Tatsache darauf hin, daß die Landwirte den Viehbestand eher nach einer hohen Ernte bemessen, als daß sie genügende Futterreserven zurückbehalten. Aufgabe der Beratung wird sein, darauf hinzuweisen, da $\beta$ es billiger ist, die Heuproduktion zu erhöhen und qualitativ zu verbessern, als das Vieh mit zugekauften Futtermitteln großzuziehen. Vermehrte Reserven an Futter sind nahezulegen.

Der wichtigste Aufwand der untersuchten Betriebe ist die Arbeit. In 1700 Betrieben waren $2732 \mathrm{AK}$ (Arbeitskräfte) tätig. Größtenteils dürfte es sich um Familienangehörige handeln. Da die 2732 Arbeitskräfte, 6153 Kühe oder 12178 GVE betreu- 
ten, hat eine AK 4,4 GVE zu pflegen, wovon die Hälfte auf Jungvieh entfällt. Dazu kommen 22 Aren Ackerland je Männereinheit. Diese Leistung je Arbeitskraft befriedigt keinesfalls. Sie ist auf die ungünstige Struktur der Betriebe, auf den saisonalen Charakter der Arbeit, den Mangel der Ausrüstung und die fehlende Technik zurückzuführen. In der Steigerung der Leistung je Arbeitskraft muß und kann die größte Aufwandeinsparung erzielt werden. Dazu ist eine innere und äußere Aufstockuny nötig. Als erstes Ziel bezeichnen wir die durchschnittliche Haltung von 6-7 GVE und den Anbau von 30 Aren Ackerland je männliche Arbeitskraft.

Der Ackerbau erhöht den Bedarf an Zugkräften und Maschinen. Diese sollten in Gebieten mit so schwachem Ackerbau auf genossenschaftlicher Basis angeschafft werden. Einachstraktoren, Seilzüge, Pflüge, Eggen, Spaten rolleggen, Kultivatoren, Rauwalzen, Sämaschinen, Düngerstreuer, Kleinbinder (an Einachstraktoren), Kartoffelbearbeitungsgeräte, Kartoffelgraber und Dreschanlagen müssen im Rahmen von Ackerbaugenossenschaften dem Einzelnen zur Verfügung gestellt werden, wenn man den Ackerbau in Graswirtschaftszonen fördern will. Dabei können einzelne Landwirte, die sich einen Einachser oder andere Maschinen anschaffen, Lohnarbeiten bei ihren Berufskollegen ausführen. Das Maschinenkapital und die Arbeitskraft wären so besser ausgenützt.

Die Ausrüstung der landwirtschaftlichen Betriebe des Vorderrheins ist ungenügend. Wohl verfügen die Ortschaften über elektrischen Anschluß für Licht und Kraft. Auch Brunnen dürften überall vorhanden sein, doch fehlt verschiedenlich die Wasserleitung und der Boiler im Hause. Manche Bauernfamilie muß auch am Hauptwohnsitz das Wasser am Brunnen holen. Es fehlt meistens an gemeinsamen Backöfen, Waschanlagen, Gefrierräumen und anderen Einrichtungen mehr, die das Leben erleichtern. Doch darüber wären Sondererhebungen nötig. An wirtschaftlichen Einrichtungen sind laut Betriebsspiegeln im Bezirk Disentis nur fünf Betriebe festgestellt, die über Silos verfügen. Dagegen gestatten einige Heubelüftungsanlagen das Nachtrocknen von unfertigem Heu. In fünf Gemeinden des Kreises Ruis wurde keine einzige Güllengrube gezählt. Im Bezirk Lugnez fehlen in sechs Gemeinden, im Bezirk Disentis in zehn die Güllengruben. Im ganzen besitzen $16 \%$ der Landwirte eine solche, während $84 \%$ der Betriebe in keinem der Ställe eine solche haben, weil nur $10 \%$ der Betriebe weniger als drei Ställe bewirtschaften und bei $84 \%$ der Betriebe mehr als 10 Parzellen vorhanden sind.

Verschiedene Gemeinden haben der Milchverwertung nur bei der Aufzucht und Eigenversorgung Beachtung geschenkt. Der verbleibende Rest an Überschußmilch wurde nur mangehaft ausgenützt. Für eine lohnende Verwertung der anfallenden Überschußmilch müssen Sammelstellen, Zentrifugierstellen oder Käsereien besser eingerichtet oder neu erstellt werden, womit aber auch das Verwertungssystem bestimmt wird. Die Wahl der Verwertungsart hat sich einerseits nach der anfallenden Milchmenge und andererseits nach den Absatzmöglichkeiten für Konsummilch zu richten. Dabei ist ratsam, auf die stets größer werdende Nachfrage nach Konsummilch in unseren Berggemeinden Rücksicht zu nehmen. Wo die neuen Transportmöglichkeiten einen wirtschaftlich verantwortbaren Zusammenzug der Tal- und Alpmilch möglich machen, verdient diese weitere Rationalisierungsmöglichkeit unsere volle Aufmerksamkeit.

Agrar-Verfassung. Am eindringlichsten zeigt aber die Auswertung der Betriebsspiegel, welche Bedeutung einer verbesserten Struktur in dem untersuchten Gebiete beizumessen ist und daß eine solche nicht nur zur Arbeitserleichterung führen kann, sondern durch die gesteigerte Leistung des Einzelnen zur wirtschaftlichen und durch die Einsparung von Mühe und Zeit zur sozialen und wohnlichen Verbesserung der bergbäuerlichen Verhältnisse beitragen kann. Trotzdem 34\% der Betriebe weniger als fünf Hektaren bewirtschaften und 47 weitere Prozent in der Größenordnung von 5-10 ha 
liegen (oder im Durchschnitt nur 4,15 GVE je Betrieb gehalten werden), sind andere Strukturprobleme wichtiger als das der Flächenvergrößerung. Nützlichere Betriebsformen erscheinen solange unmöglich, als $84 \%$ der Betriebe mehr als 10 Parzellen (ohne Alp- und Gemeinschaftsweiden) bewirtschaften. Alle Bemühungen zu einer verbesserten Düngerwirtschaft, zu einer Rationalisierung und Technisierung der Arbeit, also zur Leistungssteigerung oder Aufwandsenkung müssen an dieser Parzellierung scheitern. Ebenso wichtig ist eine verringerte Zahl von Ställen und damit eine Einschränkung des Nomadisierens der Menschen. $90 \%$ der Betriebe haben mehr als zwei, ja $22 \%$ der Betriebe haben mehr als acht Ställe. Ihre Zusammenlegung ist so wichtig, da $\beta$ die einzelnen Betriebe nicht abwarten sollten, bis das Zusammenlegungsverfahren und die Integralmelioration für ihre Gemeinde eingeleitet wird. Vielmehr ist durch nachbarlichen Austausch einzelner Grundstücke die Verringerung der Parzellenzahl anzustreben. Die Landbesitzer sind darauf hinzuweisen, da $\beta$ für Eintragungen (ins Grundbuch), die mit Bodenverbesserung oder mit Bodenaustausch zum Zweck der Arrondierung landwirtschaftlicher Betriebe zusammenhängen, keine Gebühren erhoben werden dürfen.

Die Nutzung des Gebirgslandes wird immer eine gewisse Staffelung voraussetzen. Doch sollten außerhalb der Alpkorporation, die selten mehr als zwei bis drei Staffeln bestoßen, zwei Stallungen je Betrieb nur in Ausnahmefällen überschritten werden. Die modernen Hilfsmittel, d.h. die besseren Straßen, Güllenverschlauchungen, Milchleitungen, Einachstraktoren mit Zapfwellenantrieb auf die Räder des Anhängers gestatten eine Konzentration der Wohnungen, der Stallungen, der Käsereianlagen und erlauben trotzdem eine bessere Verteilung des Düngers u. Ausnützung des Graslandes. Im übrigen werden die Schwierigkeiten bei den Transportbedingungen des Gebirges durch verbesserte Struktur stärker vermindert als durch irgendwelche Mechanisierung. Wenn der zwei volle Arbeitskräfte beschäftigende Betrieb auch im Vorderrheintal zum Ideal er. hoben würde, müßte derselbe ohne Nebenerwerb und Spezialnutzung 12 GVE, 60 Aren Ackerland und für die Winterarbeit etwas Wald oder Waldnutzung umfassen. Wir glauben aber, daß der kleinere Betrieb mit Nebenerwerbsmöglichkeiten vor allem aus bevölkerungspolitischen Gründen, d. h. zur Festigung der Dorfkultur im Berggebiet erhalten bleiben muß.

Die Steigerung der Erträge, also die Intensivierung der Betriebe scheint also unbcdingt erforderlich. Sie soll aber zur Einsparung an Kraftfutter-, Stroh-, Heuzukäufen, Gebäudekapital, Transportmitteln usw. führen. Die durch eine verringerte Gebäudezahl am Gebäudekapital ermöglichten Einsparungen sollten unbedingt zu Investitionen und zur nötigen Verbesserung des ländlichen Wohnkomfortes und dem Ausbau der verbleibenden Stallungen (Düngergewinnung) dienen. Eine Extensivierung, die zum einseitigen Großbetriebe führen würde, ist weniger aus agrartechnischen als aus volkswirtschaftlichen und erst recht aus bevölkerungs- und kommunalpolitischen Gründen abzulehnen. Sie würde zu einem verstärkten Kontrast zwischen der Finanzkraft von bergbäuerlichen und Flachlandgemeinden führen, soda $\beta$ die ersteren ihren Aufgaben nicht mehr gerecht werden könnten.

Die Entvölkerung des Berggebietes würde beschleunigt. Die Leistungssteigerung je AK sollte jedoch nicht nur durch die Produktionssteigerung, sondern wie es in diesem Bericht angedeutet wurde, auch durch die Ausdehnung des Ackerlandes und eine erhöhte Selbstversorgung mit Kraftfutter und Stroh, also durch Aufwandverringerung, vermehrte Schweine- und Geflügelhaltung und die inneren Aufstockungen der Betriebe erreicht werden. Diese Vermehrung des Ackerbaus wird die ausgeglichenere Ausnützung der Arbeitskräfte bringen und durch die breitere Grundlage der Bodennut. zung das Risiko verteilen. Wir glauben, daß der Ackerbau im Vorderrheintal wesentlich gesteigert werden könnte und setzen das Ziel je GVE für die Getreidefläche in der günstigeren Zone (Gemeinden: Felsberg, Tamins, Bonaduz, Ems, Rhäzüns) 10 Aren, 
ev. auch 20 Aren je nach den örtlichen Verhältnissen, in der zweiten Zone (Gemeinden des Kreises Ilanz, Flims, Trins und das Rheingebiet bis Somvix und Disentis sowie die bestsituierten Gemeinden des Kreises Lugnez) 5-10 Aren, ev. bis 20 Aren je GVE, in der dritten Zone (Gemeinden Vrin, Surin, Medels, Tavetsch und Mompemedel) 1-5 Aren je GVE. Das Safiental und Vals können nur unbedeutend Getreide anbauen. Die Bearbeitung der Ackerflächen würde auch zur Verbesserung der Grasnarbe beitragen, sodaß der ganze Betrieb davon profitiert.

Zur strukturellen Verbesserung gehört eine verlängerte Weidezeit. Die Stallhaltung ist im Vergleich zur Weidezeit im Gebirge besonders teuer. Wenn man schon im Flachland längere Weidezeit anstrebt, so sind deren Vorteile im Gebirge noch größer. Verschiedene Maßnahmen ermöglichen eine Verlängerung des Austriebes. Die Weiden, die im Frühjahr als erste dem Austrieb dienen sollen, sind schon im Herbst dafür vorzubereiten. Man darf sie nicht bis zuletzt beweiden, sondern muß durch Düngung und Ruhe vor dem Schneefall eine Stärkung des Graswuchses erreichen. Auf solchen Flächen wird der Austrieb im Frühjahr früher möglich sein. Weiter sollten Galt- und Jungvieh getrennt von den Kühen und früher ausgetrieben werden. Dieser Austrieb könnte auf den Maiensäßen, oder Voralpen, ähnlich wie auf der eigentlichen Alp kooperativ erfolgen und damit wäre wesentlich Arbeitsaufwand einzusparen.

Der Weideplan hat also drei Punkte zu verfolgen:

1. Stärkere Trennung von Kühen und Galtvieh, nicht nur auf der Alp, sondern überall, wo genossenschaftliche Haltung möglich ist (Mittel zur Arbeitseinsparung).

2. Längere Weideperioden zur Verbilligung der Fütterung.

3. Geringerer Wechsel der Staffeln, namentlich bei den Kühen im Interesse des vereinfachten Betriebes.

\section{A US B LI C K}

Zusammengefaßt zeigen die Erhebungen, daß die Produktionsleistung der Betriebe und damit ihr Ertrag durch Verstärkung des Ackerbaus, durch bessere Düngung und durch Wiesenpflege auf eigener Futterbasis und durch rationellere Arbeit gehoben werden kann. Voraussetzungen für eine bessere Düngertechnik, Arbeitsproduktivität, Aufwandsenkung sind aber die Strukturverbesserungen, also Zusammenlegung (nicht nur der Talbetriebe), Verminderung der Staffelzahl, der Ställe und Scheunen. Weitere Voraussetzung ist auch ein verstärktes Zusammenarbeiten der in der Gemeinde lebenden Landwirte, beim Weiden, beim Ackerbau, bei Transporten, bei der Produktionsverwertung und bei der Beschaffung von Hilfstoffen, um den Aufwand in einem erträglichen Rahmen zu halten. All das sollte auf genossenschaftlicher Basis erfolgen. Neben der Arbeit auf dem landwirtschaftlichen Betrieb muß für den Bergbauer die Möglichkeit bestehen, sein Einkommen durch Nebenerwerb zu steigern. Es wäre von Nutzen, über diese mehr das soziale als das betriebswirtschaftliche Problem berührenden Fragen eine Sonderuntersuchung zu machen.

Der Landwirtschaft verbleiben somit im Vorderrheintal mannigfache Aufgaben. Ihre Lösung verbürgt indessen nicht nur wesentlich verbesserte Lebensverhältnisse der Bergbevölkerung, sie wird auch Gesicht und Charakter der Agrarlandschaft (Reduktion der Zahl der Gebäulichkeiten und Parzellen, Vermehrung der Ackerflächen usw.) zu ihrem Vorteil zu wandeln vermögen. Darum sollte hiefür alle Energie eingesetzt werden.

\section{LITERATUR}

Casurtr, C. P.: Beitrag zur Lösung der Bergbauernfrage. Chur 1954. - Il pur seprepara per la primavera. Cuera 1960 . - und RAUCH, E.: Bericht über die Landwirtschaft im Vorderrheintal auf Grund der Betriebsspiegel 1958/59. Dokument No 84 der Schweiz. Vereinigung zur Förderung der Betriebsberatung in der Landwirtschaft. Küsnacht/ZH 1961. STRUEBY, A.: Die Alpwirtschaft im Kt. Graubünden. Bern 1909. FrÜH, J.: Geographie der Schweiz. III. Die Landschaften. St. Gallen 1938. Gutersohn, H.: Geographie der Schweiz II. Alpen I. Bern 1961. Vincenz, G. C.: Bewirtschaftungs- und Ertragsverhältnisse von Bündner Alpen. Winterthur 1960. 


\title{
GEOGRAFIA AGRARA DALLA VALLADA DIL REIN ANTERIUR
}

L'Uniun Svizzera per promoziun della cussegliaziun purila ha daquort intercuriu, sin fundament da sias retschercas en las valladas muntognardas, la vallada dil Rein anteriur. Ils resultads leu constatai ein era per la politica agrara da gronda valeta, aschia ch' ei gli' ei vegniu orientau sur tala en la lavur presenta. En la vallada dil Rein anteriur anflein nus (1955) 3162 menaschis purils, per 2242 purs fuva il far il pur la clamada prinzipala. La grondezia media dils menaschis munta rodun 5 hectaras. Las intradas darivan per $90 \%$ dalla tratga da biestga. Il spazi dad ér contonscha mo darar $10 \%$ dil funs dil menaschi. Per anflar existenza sufizienta ston ins fetg savens aunc encurir ina intrada dasperas. A beinhiars menaschis porta la tratga d'animals manedels ina buna intrada sper la tratga da biestga. Mo sin fundament da quella, en uniun cun ina excelenta tratga da biestga armentiva, eis ei pusseivel a biars purs da saver semantener e prosperar. La pusseivladad d'ina pli vasta producziun saves vegnir segirada tras in'augmentaziun digl areal dad ér. Leutier drova ei en general ina pli intensiva cultivaziun dil funs combinada cun ina beinponderada razionalisaziun dalla lavur. Emprema premissa per contonscher quei intent ei ina radicala midada dalla structura da menaschi, sco arundaziun dil funs, sminuaziun ed ingrondaziun dallas singulas parzellas da prau, aclas e cuolms, reducziun dils nuegls e clavaus da fein. Ultra digl allegau fus era ina pli stretga collaboraziun denter ils purs nezessaria, spezialmein alla cultura dad érs, pesculaziun, transports e surtut era alla cumpra dals mieds da producziun ed alla vendita dils agiens products. Tut quei fus d'organisar sin fundament da societad. Ultradaquei stues e fus ei pusseivel d'augmentar il gudogn dasperas (lavur d'uaul, industria d'jasters e.a.v.). All'agricultura della vallada dil Rein anteriur tuca ei da sligiar els proxims onns bia pensums e tals da diversas natiras. La sligiaziun da quels spegierscha buca mo ina megliuraziun dallas relaziuns d'esser e semantener al singul carstgaun. Era il caracter da nossa tiara purila vegn a profitar lunderora e semidar per avantatg dall'entira populaziun.

\section{LAC DE TSEUZIER}

\section{HeinRich Gutersohn}

\author{
Mit Farbtafel
}

Zur Rhone stoßen auf Walliser Boden von rechts her nur verhältnismäßig kurze Täler mit entsprechend kleineren Flüssen. Einer der längsten unter ihnen ist La Liènc. Ihre Quellbäche entspringen zwischen Wildhorn und Wildstrubel, also im Gebiet der Rawil-Senke. Von den Gletschern dieser beiden Gebirgsstöcke kommt der Liène verhältnismäßig viel Wasser zugute, und es war deshalb naheliegend, daß sich die Elektrizitätswirtschaft dieses Quellgebiet zu nutzen bestrebte. Hiefür bot auch die Oberflächengestaltung günstige Voraussetzungen. Montagne du Rawil, in rund $1700 \mathrm{~m}$ gelegen, ist ein Gebirgsbecken, in welchem sich früher die Alp Tseuzier ausbreitete. Talauswärts ist es durch eine mit einer Felsenge betonten Stufe abgeschlossen, die sich vorzüglich für den Einbau einer Talsperre eignete. In den Jahren 1953-58 wurde die Schlucht durch die $155 \mathrm{~m}$ hohe und $265 \mathrm{~m}$ lange Staumauer abgedämmt. Dahinter ist der Lac de Tseuzier, ein $1 \frac{1}{2} \mathrm{~km}$ langer, $50 \mathrm{Mio} \mathrm{m}^{3}$ fassender Stausee mit maximalem Wasserspiegel in $1777 \mathrm{~m}$ entstanden. Er hat das Becken von Montagne du Rawil mit der Alp Tseuzier überflutet. Das Nutzwasser gelangt durch die anschließende Druckleitung in die in $920 \mathrm{~m}$ im Talweg der Liène gelegene Kavernenzentrale Croix, von hier aus durch einen weiteren Stollen und anschließenden Druckschacht in die Zentrale St-Léonard im Rhonetal, womit ein Gefälle von $1276 \mathrm{~m}$ für eine Stromerzeugung von rund 180 Mio $\mathrm{kWh}$ ausgenutzt wird.

Der Stausee vermittelte der Landschaft zweifellos einen Gewinn, denn anstelle einer eher kargen und etwas düsteren Alpweide bietet sich dem Auge des Besuchers nun ein romantischer Bergsee dar, an dessen Ufer noch kleine Weiden grenzen, und von dem aus dunkel bewaldete Bergflanken aufstreben, im Hintergrund überragt von den weißen Gipfelgestalten der Berneralpen. Heute wird der See gerne von Wanderern aufgesucht. Aber auch dem Automobilisten ist der Besuch leicht gemacht, denn mit dem Bau des 